\title{
THE PARAMETRIC \\ HIGH-FIDELITY-GENERALIZED-METHOD-OF-CELLS (PHFGMC) MICROMECHANICAL MODEL FOR COMPRESSION FAILURE OF FRP COMPOSITES
}

\author{
Ido Meshi $^{1}$, Uri Breiman ${ }^{2}$ AND Rami Haj-Ali ${ }^{3}$ \\ ${ }^{1}$ School of Mechanical Engineering, Tel-Aviv University \\ Ramat Aviv 69978, Israel \\ idomeshi@mail.tau.ac.il \\ ${ }^{2}$ School of Mechanical Engineering, Tel-Aviv University \\ Ramat Aviv 69978, Israel \\ uribreiman@mail.tau.ac.il \\ ${ }^{3}$ School of Mechanical Engineering, Tel-Aviv University \\ Ramat Aviv 69978, Israel \\ rami98@tauex.tau.ac.il
}

Key words: PHFGMC, micromechanics, multiscale, mesoscale

\begin{abstract}
A multiscale model based on finite element (FE) and the Parametric High-Fidelity-GeneralizedMethod-of-Cells (PHFGMC) micromechanical model was formulated and implemented to solve the compression problem in unidirectional IM7/977-3 carbon epoxy composite. The nonlinear PHFGMC governing equations were obtained from a two-layered (local-global) virtual work principle and solved using a incremental-iterative formulation. In addition, the semi-analytical modified Lo and Chim failure criterion (based on the buckling of Timoshenko's beam) for unidirectional fiber-reinforced composite materials under compression [1] was adopted and combined with the FE-PHFGMC multiscale model. In this study, the criterion was employed for the general case of a multi-axial loading state accompanied with a nonlinear polymeric matrix behavior, where the local and thus effective properties of the composite change continuously throughout the loading path. Therefore the predicted lamina strength was incrementally reevaluated. In the present model, the use of the nonlinear constitutive model RambergOsgood was used for the matrix media and a linear-elastic transversely-isotropic law for the fiber, as common for carbon fibrous composites. This extends the existing criterion to account for the material microstructure with a refined parametric discretization, as well as the effect of a nonlinear constitutive law. The advantage of the proposed model is to predict the compressive damage (kink band formation and its width) and the compressive strength (within $11 \%$ of experimental data).
\end{abstract}

\section{INTRODUCTION}

According to the U.S. Department of commerce, advanced composite structures are at the forefront of innovative solutions for the challenges facing leading industries and communities, e.g. rebuilding 


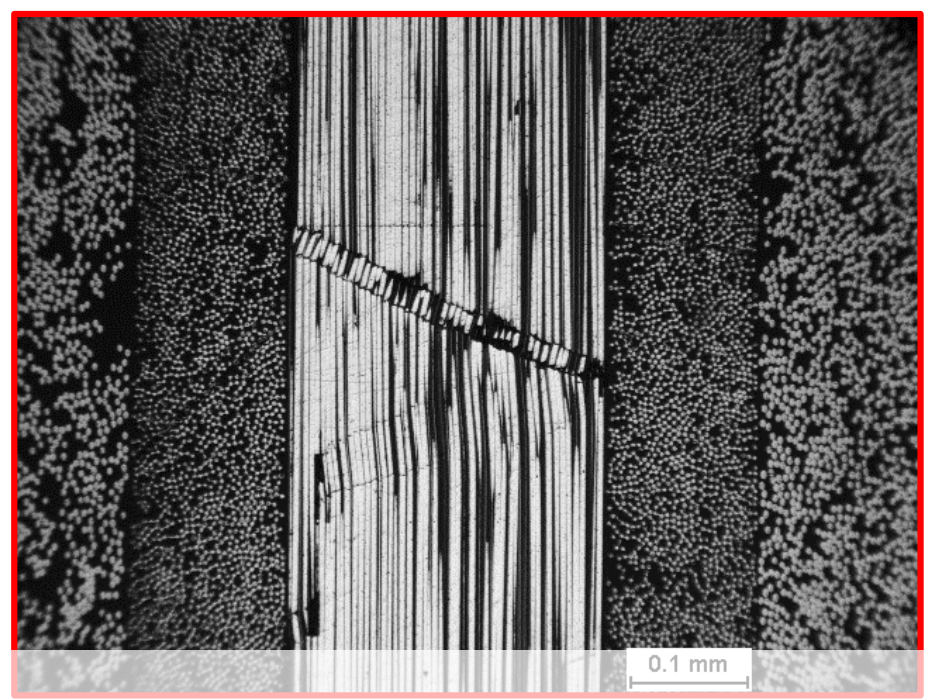

Figure 1: Kink band in a carbon reinforced composite IM7/977-3 under compression

old infrastructure, building new climate resilience public utilities, and improving the fuel-efficiency of airplanes and vehicles [2]. The main advantage of composites is their light weight and increased strength compared to traditional materials.

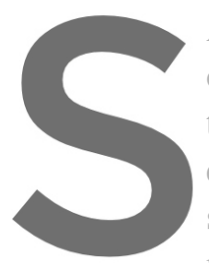

Accurate local and globa ous loading conditions, ticular, this study focused epoxy composite. This sured in experiments is
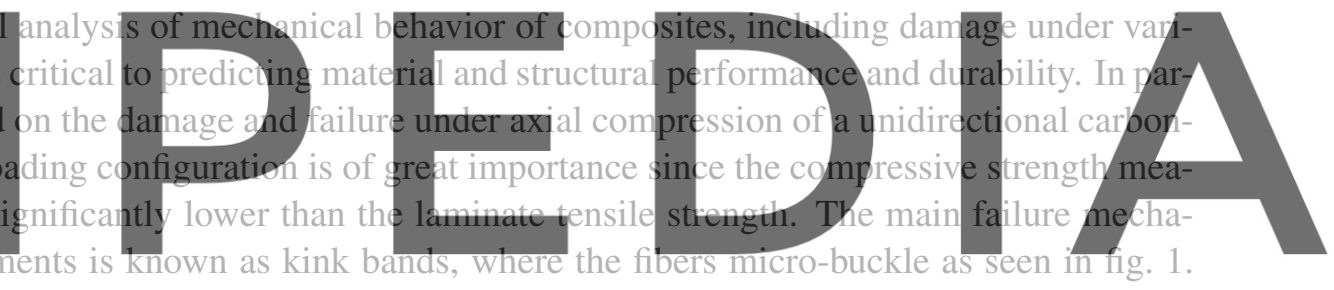

Early works considered the fiber buckling (and breakage due to bending) to be the driver of the kink

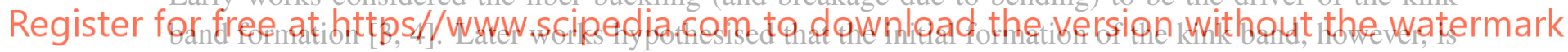

a result of damage in the matrix phase that causes instability after which the fibers buckle. The matrix damage is atributed to axial shear stresses that are concentrated around material inhomogeneities like fiber waviness $[5,6]$.

The kink band width is found to be from 10 to 25 times the fiber diameter [7, 8], and cannot be captured by a two dimensional representation of the microstructure (as in the case of the current PHFGMC model). This larger length-scale requires a mesoscale analysis, where at least several fibers are included in the domain, in all three dimensions, to capture the fiber-matrix interaction that produces the stress field, damage, instability, and ultimately, the kink band. Many works modeled the mesoscale problem for various material systems and microstructures $[9,10,11]$ among others. This numerical analysis is usually carried out by explicitly modeling the fibers and matrix in a finite element (FE) model, which results in a very large number of degrees-of-freedom (DOF) necessary to bridge the gap between the micro and meso scales. Moreover, this approach is impractical for larger specimens or part assemblies. The multiscale approach proposed in this study aims at reducing the number of variables while accurately predicting the compressive strength and kink band formation. 
To this end, a multiscale FE-PHFGMC analysis was formulated and implemented for compression loading. The nonlinear PHFGMC micromechanical model [12] is a dedicated micromechanical method, extending its HFGMC predecessor. The microstructure is discretized into volumetric subcells that form the RUC and representing the composite's periodic microstructure. The PHFGMC model requires less DOF than a comparable FE model due to a combination of factors [13]. In the current formulation, the linearized governing equations are obtained from a virtual work principle applied to both the micro and macro/meso scale variables and solved using a new incremental-iterative formulation. The proposed formulation yields an overall symmetric system of equations and also enables the implementation of advanced numerical stabilization methods available in current FE programs. Predictions of the FEPHFGMC for damage initiation, its evolution, and the overall stress-strain response are shown to be in good agreement with experimental data.

\section{METHODS}

The multiscale algorithm implemented in this work used a FE model at the macro/meso scale and the PHFGMC model at the microscale. Note that the fibers and matrix are modeled explicitly only at the microstructure level and not at the mesoscale. This allows for a relatively coarse mesh at the mesoscale and a finer mesh only at the microscale problem. During the solution procedure, the strain field at the mesoscale (far-field) is passed to the microscale at every material (integration) point as the applied boundary conditions of the micromechanical problem. The PHFGMC model is then used to solve this problem resulting in the local fields and damage state in the microstructure. The local stress field is homogenized and it is passed back to the higher scale along with the RUC effective stiffness. After assembly, the FE incremental-iterative nonlinear solver is called.

2.1 Governing equations of the PHFGMC model

In the PHFGMC micromechanical model formulation for doubly
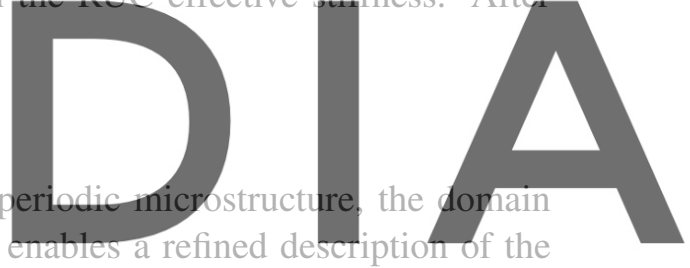

microstructure and a higher resolution of the calculated fields. A standard linear mapping using bi-

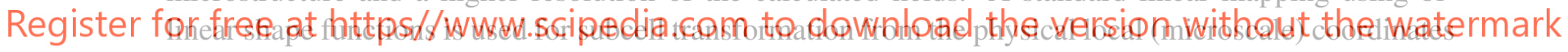

$\left(y_{2}, y_{3}\right)$ to the uniform parametric coordinate system $(r, s)$, where the calculations at the subcell level are carried out [14] (see fig. 2). The displacement vector in a random subcell ( $\beta$ ) is given by [12]:

$$
\begin{aligned}
\mathbf{u}^{(\beta)} & =\boldsymbol{\varepsilon}_{0} \cdot \mathbf{x}+\boldsymbol{W}_{0}^{(\beta)}+\frac{1}{2}\left(\boldsymbol{W}_{2}^{(\beta)}-\boldsymbol{W}_{4}^{(\beta)}\right) r+\frac{1}{2}\left(\boldsymbol{W}_{3}^{(\beta)}-\boldsymbol{W}_{1}^{(\beta)}\right) s \\
& +\frac{1}{4}\left(\boldsymbol{W}_{2}^{(\beta)}+\boldsymbol{W}_{4}^{(\beta)}-2 \boldsymbol{W}_{0}^{(\beta)}\right)\left(3 r^{2}+r s-1\right) \\
& +\frac{1}{4}\left(\boldsymbol{W}_{1}^{(\beta)}+\boldsymbol{W}_{3}^{(\beta)}-2 \boldsymbol{W}_{0}^{(\beta)}\right)\left(3 s^{2}+r s-1\right)
\end{aligned}
$$

where the first term is the displacement associated with a linear far-field strain field of the macro scale $\mathbf{x}$ and the terms that follow form the quadratic perturbation field in the micro scale $\mathbf{y}$. The microvariable vectors are $\left(\boldsymbol{W}_{1}, \boldsymbol{W}_{2}, \boldsymbol{W}_{3}, \boldsymbol{W}_{4}, \boldsymbol{W}_{0}\right)$, where $\boldsymbol{W}_{i}(i=1,2,3,4)$ represent the average displacement on the subcell faces, and $\boldsymbol{W}_{0}$ is an additional microvariable vector. 
(a)

(b)

(c)
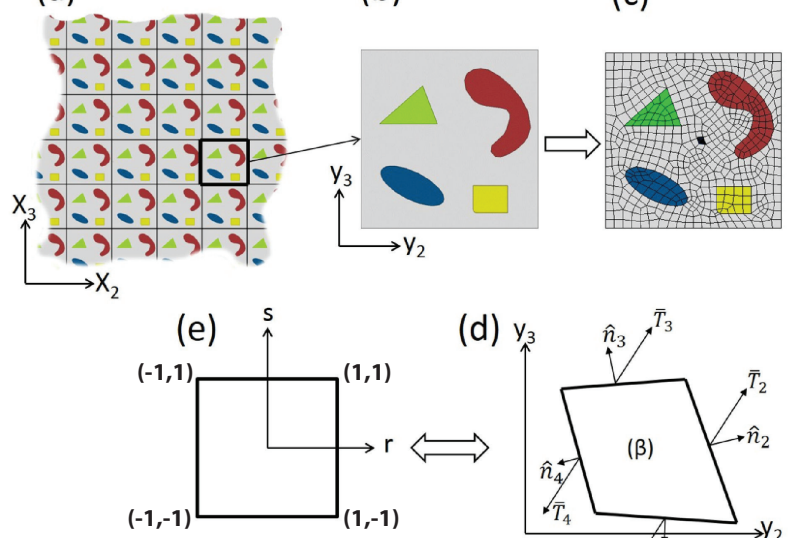

(d) $y_{3}$

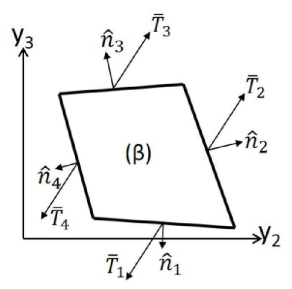

Figure 2: Adapted from [15]: Schematic representation of doubly periodic composite in the PHFGMC model. The composite (a) is made of multiple RUC's (b). The composite is represented by a single RUC (c) divided into an arbitrary number of subcells under applied remote strain and periodic BC. Each subcell is mapped from the physical to the parametric domain (e),(d)

For a random subcell $(\beta)$, the external and internal average virtual work balance is expressed by:
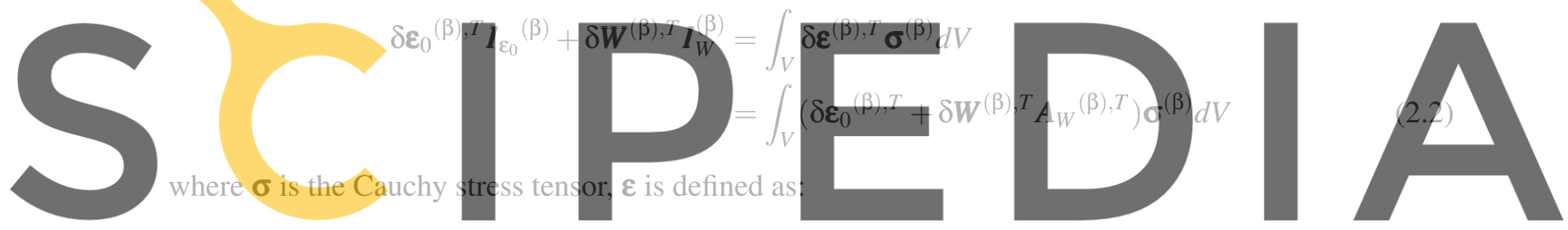

$$
\varepsilon^{(\beta)}=\varepsilon_{0}+A_{W}^{(\beta)} W^{(\beta)}
$$

Register for free at https//www.scipedia.com to download the version without the watermark with $\boldsymbol{A}_{W}^{P}$ containing linear functions of the parametric coordinates and the Jacobian of the parametric mapping. The generalized internal resisting force vector of the subcell can be identified with its two parts as:

$$
\boldsymbol{I}_{\varepsilon_{0}}^{(\beta)}=\int_{V^{(\beta)}} \boldsymbol{\sigma}^{(\beta)} d V \quad ; \quad \boldsymbol{I}_{W}^{(\beta)}=\int_{V^{(\beta)}} \boldsymbol{A}_{W}{ }^{(\beta), T} \boldsymbol{\sigma}^{(\beta)} d V
$$

By using the generalized Hooke's law for infinitesimal strains with eq. (2.3), the stress tensor can be described in terms of the microvariables and the far-field infinitesimal strain:

$$
\boldsymbol{\sigma}^{(\beta)}=\boldsymbol{C}^{(\beta)} \boldsymbol{\varepsilon}^{(\beta)}=\boldsymbol{C}^{(\beta)}\left(\boldsymbol{\varepsilon}_{0}+\boldsymbol{A}_{W}^{(\beta)} \boldsymbol{W}^{(\beta)}\right)
$$

The obtained equations can be formulated in a matrix form:

$$
[\boldsymbol{K}]\{\hat{\mathbf{u}}\}=\{\boldsymbol{I}\}
$$

The algorithmic stiffness matrix that relates the overall generalized force vector, $\boldsymbol{I}=\left(\boldsymbol{I}_{W}, \boldsymbol{I}_{\varepsilon_{0}}\right)$, and the overall variables of the subcells, $\hat{\mathbf{u}}=\left(\boldsymbol{W}, \boldsymbol{\varepsilon}_{0}\right)$, is obtained by assembly of the stiffness matrices of all the 
subcells. The subcell stiffness is expressed by:

$$
\boldsymbol{K}^{(\beta)}=\left[\begin{array}{cc}
\int_{V} \boldsymbol{A}_{W}^{T} \boldsymbol{C} \boldsymbol{A}_{W} d V & \int_{V} \boldsymbol{A}_{W}^{T} \boldsymbol{C} d V \\
\int_{V} \boldsymbol{C} \boldsymbol{A}_{W} d V & \int_{V} \boldsymbol{C} d V
\end{array}\right]^{(\beta)}=\left[\begin{array}{cc}
\boldsymbol{K}_{W W} & \boldsymbol{K}_{W \varepsilon_{0}} \\
\boldsymbol{K}_{\varepsilon_{0} W} & \boldsymbol{K}_{\varepsilon_{0} \varepsilon_{0}}
\end{array}\right]^{(\beta)}
$$

Note that indeed the stiffness matrix of the PHFGMC is symmetric. The second-order Gaussian quadrature rule using 2-by-2 integration points is suitable for numerically evaluating the above integrals since the integrands are either linear or quadratic functions. Further, the traction continuity between neighboring subcells is satisfied in an average sense by the weak formulation. The displacement continuity and periodicity could be easily achieved by associating the microvariables of shared faces with a single active microvariable in the overall system of equations.

Periodicity constraints are applied to the microstructure boundary, such that the displacement of two opposite points on opposite faces are set equal:

$$
\mathbf{u}(\mathrm{y})=\mathbf{u}(\mathrm{y}+\mathbf{L})
$$

where $\mathbf{L}$ is a spatial vector in the periodicity direction with a magnitude of one periodic RVE length (in that direction). The implementation in the PHFGMC is done pair-wise in an average sense, which leads to the constraints on the microvariables. Namely, the average displacement on the $k$ th face of some random subcell $(\beta)$ using the aforementioned linear mapping is given by [16]:
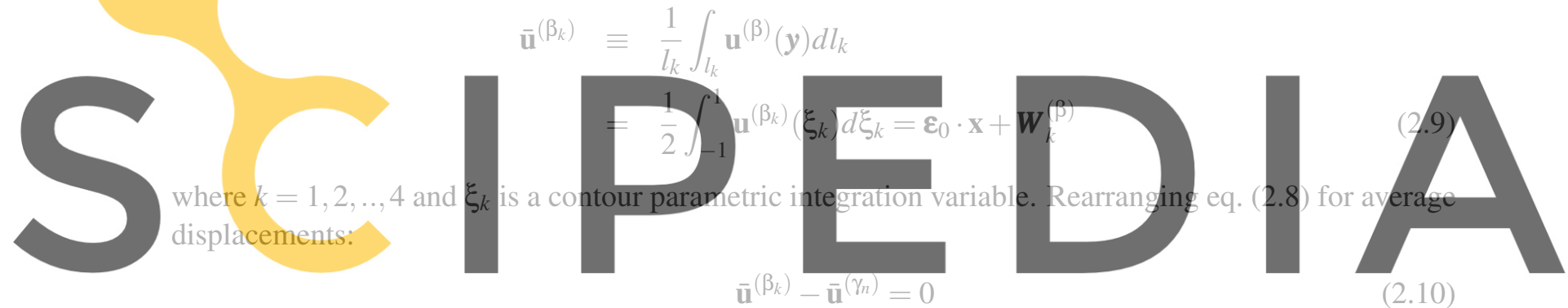

Register for free at https//www.scipedia.cow tho downoload the version without the (מatermark

where $\beta$ and $\gamma$ are indices of any pair of periodic subcells, and $k, n$ are the relevant faces of each subcell.

\section{Incremental-iterative procedure}

In the case of nonlinear material response, the Newton incremental-iterative method is employed. The following nonlinear derivations assume incremental and tangential variables similar to the total-variables previously derived in the linear part. The equilibrium residual of the entire system of equations can be expressed in a general manner by:

$$
\boldsymbol{R} \equiv \boldsymbol{I}-\boldsymbol{P}=\left\{\begin{array}{c}
\int_{V} \boldsymbol{A}_{W}^{T} \boldsymbol{\sigma} d V \\
\int_{V} \boldsymbol{\sigma} d V
\end{array}\right\}-\left\{\begin{array}{c}
\boldsymbol{P}_{W} \\
\boldsymbol{P}_{\varepsilon_{0}}
\end{array}\right\}
$$

where $\boldsymbol{I}$ is the internal force vector and $\boldsymbol{P}$ is the external force vector (and $\boldsymbol{P}_{W}, \boldsymbol{P}_{\varepsilon_{0}}$ are the external load applied to the microscale, macroscale, respectively). Under equilibrium $\boldsymbol{R}=\mathbf{0}$ and by using Newton's method:

$$
\frac{\partial \boldsymbol{R}}{\partial \hat{\mathbf{u}}} \Delta \hat{\mathbf{u}}=-\boldsymbol{R}
$$


Under the assumption that the external load is independent of the generalized displacement and from eq. (2.6) the global stiffness matrix is:

$$
\frac{\partial \boldsymbol{R}}{\partial \hat{\mathbf{u}}}=\frac{\partial \boldsymbol{I}}{\partial \hat{\mathbf{u}}} \equiv \boldsymbol{K}
$$

In the current formulation, the static condensation method is performed, where the equations associated with the applied remote displacement field are eliminated. Noting that the external load applied on the microscale $\boldsymbol{P}_{W}$ is zero (since all the external loads are applied on the macroscale) ultimately leads to the incremental linearized symmetric system of equations solving for the microvariables (from $n$ to $n+1$ ):

$$
\boldsymbol{K}_{W W}^{n} \Delta \boldsymbol{W}^{n+1}=-\boldsymbol{I}_{W}^{n}-\boldsymbol{K}_{W \varepsilon_{0}}^{n} \Delta \boldsymbol{\varepsilon}_{0}^{n+1}
$$

where the constitutive tangent matrix $\boldsymbol{C}^{(\beta), n}$ is used to evaluate $\boldsymbol{K}_{W W}^{n}, \boldsymbol{K}_{W \varepsilon_{0}}^{n}$.

For a full Newton incremental-iterative scheme, iterations are performed in each increment in the following manner (from $i$ to $i+1$ ):

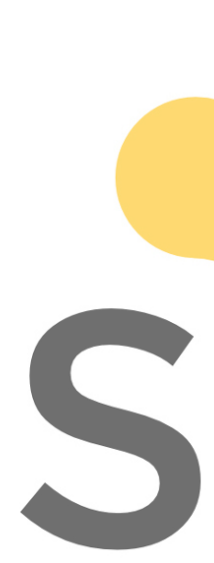

$$
\begin{aligned}
\boldsymbol{K}_{W W}^{i} \Delta \widetilde{\boldsymbol{W}}^{i} & =-\boldsymbol{I}_{W}^{i}-\boldsymbol{K}_{W \varepsilon_{0}}^{i} \Delta \widetilde{\boldsymbol{\varepsilon}}_{0}^{n+1} \\
\Delta \boldsymbol{W}^{n+1} & =\Delta \boldsymbol{W}^{n+1}+\Delta \widetilde{\boldsymbol{W}}^{i} \\
\Delta \widetilde{\boldsymbol{\varepsilon}}_{0}^{n+1} & = \begin{cases}\Delta \boldsymbol{\varepsilon}_{0}^{n+1}, & i=1 \\
0, & i \neq 1\end{cases}
\end{aligned}
$$

\section{Eiffective properties}

Once the set of equation

are calculated using eqs

$G^{(\beta), t}$ can be readily es

strain increment $\Delta \varepsilon_{0}$

arbitrary subcell $(\beta)$ :

$$
\begin{aligned}
& \text { Register for free at https//www.scipedia.com to download the version without the watermark } \\
& =\Delta \boldsymbol{\varepsilon}_{0}+\frac{1}{2 V^{(\beta)}} \int_{A^{(\beta)}}\left(\Delta \boldsymbol{u}^{(\beta)} \otimes \boldsymbol{n}^{(\beta)}+\boldsymbol{n}^{(\beta)} \otimes \Delta \boldsymbol{u}^{(\beta)}\right) d A \\
& =\Delta \boldsymbol{\varepsilon}_{0}+\frac{1}{2 V^{(\beta)}} \sum_{k=1}^{6} A^{\left(\beta_{k}\right)}(\Delta \overline{\boldsymbol{u}} \otimes \boldsymbol{n}+\boldsymbol{n} \otimes \Delta \overline{\boldsymbol{u}})^{\left(\beta_{k}\right)} \equiv \boldsymbol{G}^{(\beta), t}: \Delta \boldsymbol{\varepsilon}_{0}
\end{aligned}
$$

where $V^{(\beta)}$ is the volume of subcell $(\beta), \Delta \boldsymbol{u}^{(\beta)}$ is the displacement field increment, $\boldsymbol{n}^{(\beta)}$ is the normal to the subcell boundary $A^{(\beta)}$, and each face of the subcell is noted by $\beta_{k}$.

The tangent effective constitutive tensor $\boldsymbol{C}^{*, t}$ that relates the global average stress to the applied strain can be determined from:

$$
\boldsymbol{C}^{*, t}=\frac{1}{V_{\text {total }}} \sum_{\beta=1}^{N_{\mathrm{sc}}} \int_{V^{(\beta)}} \boldsymbol{C}^{(\beta), t} \boldsymbol{G}^{(\beta), t} d V
$$

where $V_{\text {total }}$ is the total volume of the domain, $\boldsymbol{C}^{(\beta), t}$ is the local tangent stiffness matrix of subcell $\beta$, and $N_{\text {sc }}$ is the total number of subcells. 


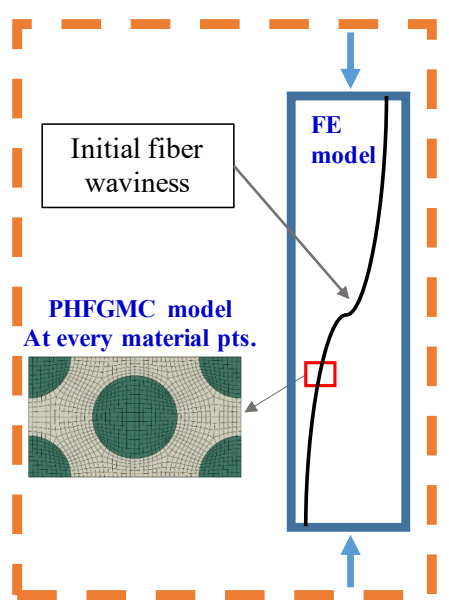

Figure 3: Schematic illustration of the FE-PHFGMC multiscale model

\subsection{Mesoscale FE-PHFGMC model setup and configuration}

The analysis was carried out in Abaqus FEA 2018 software. In fig. 3 a schematic illustration of the FE-PHFGMC is presented. The 3D mesoscale FE model was used with large deformation, corotational formulation to solve for the large rotations near the kink band edges. The fiber initial waviness was modeled using the following trigonometric function:
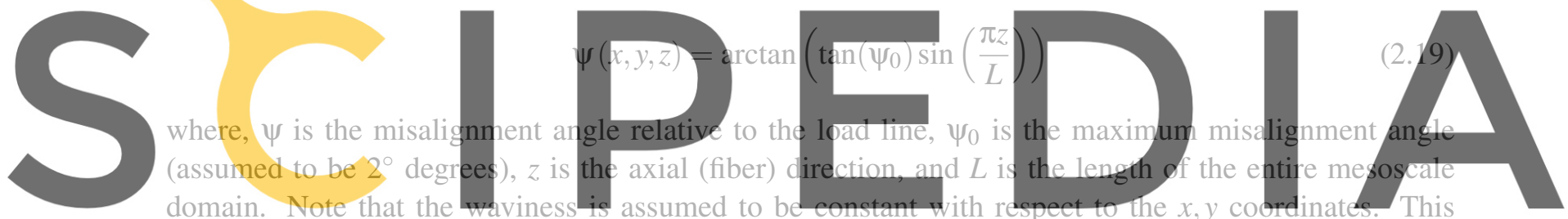

formula was implemented using the ORIENT user subroutine [17]. The overall mesoscale dimensions

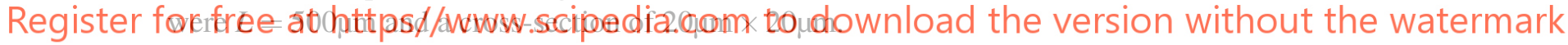

The element type was the C3D8R, a general purpose linear brick element, with reduced integration (one integration point). İts size at the gauge area was $10 \mu \mathrm{m}$ in all directions. The element size is smaller than the expected kink band width for a sufficient spacial resolution. The boundary conditions were applied only at the mesoscale. The microstructure is geometrically represented by a $2 \mathrm{D}$ hexagonal array, however, note that the PHFGMC formulation and solution are in all three dimensions. Periodicity in the FE as well (except for the axial direction) to avoid macro buckling by using *EQUATION constraint. Viscus regularization was included to address the instability (automatic stabilization with dissipated energy fraction set to 0.005). The automatic time incrementation was set to a maximum increment of 0.025 .

The PHFGMC micromechanical model was implemented as a UMAT user subroutine that is called at every macroscopic material point. The composite investigated in this study was IM7/977-3 unidirectional carbon-epoxy composite. The fiber was assumed to be anisotropic linear elastic with the properties given in table 1. The matrix constitutive law was Ramberg-Osgood J2 plasticity [18] to capture both the elastic and damage responses (properties in table 2). The one-dimensional representation of this constitutive 
law is given by:

$$
E \varepsilon=\sigma+\alpha\left(\frac{|\sigma|}{\sigma_{0}}\right)^{(n-1)} \sigma
$$

where, Young's modulus, $E$, Poisson's ratio, $v$, Yield stress, $\sigma_{0}$, Exponent, $n$, Yield offset, $\alpha$. Note that this is an implicit equation with respect to the stresses, which has to be solved iteratively. (For the generalized 3D form see [19]). In addition, the modified Lo and Chim failure criterion [20,1] was applied for the entire material point (after the microscale problem is solved) to control the kink band width:

$$
\sigma_{l c}=\frac{G_{A}^{*}}{1.5+12\left(\frac{5.195}{\pi}\right)^{2}\left(\frac{G_{A}^{*}}{E_{A}^{*}}\right)}
$$

where, $\sigma_{l c}$ is the critical axial stress predicted by the model, $G_{A}^{*}$ and $E_{A}^{*}$ are the effective longitudinal modulus and effective axial shear modulus, respectively. Note that the $E_{A}^{*}$ and $G_{A}^{*}$ values could vary throughout the mesoscale based on the microscale solution at each material point. This implies that the critical value is not constant in all points and, in fact, lower in damaged areas.

Table 1: PHFGMC model: The fiber linear elastic anisotropic properties

\begin{tabular}{cccccc}
\hline$E_{11}, E_{22}(\mathrm{MPa})$ & $E_{33}(\mathrm{MPa})$ & $v_{12}, v_{13}$ & $v_{23}$ & $G_{12}(\mathrm{MPa})$ & $G_{13}, G_{23}(\mathrm{MPa})$ \\
\hline 18500 & 265000 & 0.55 & 0.02094 & 6000 & 13000 \\
\hline
\end{tabular}
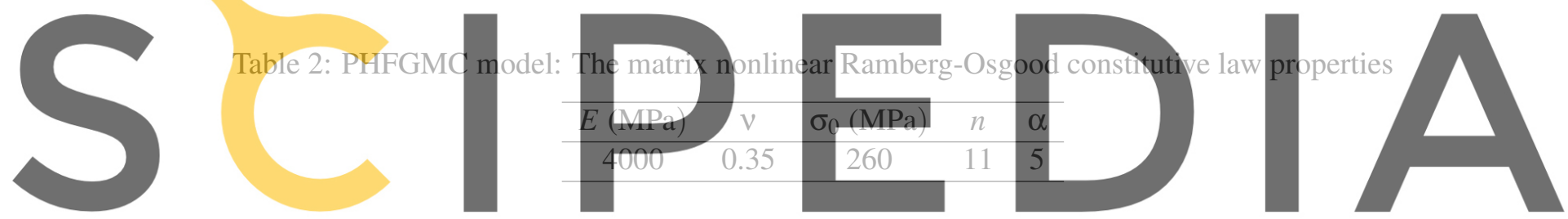

\section{Results}

Register for free at https//www.scipedia.com to download the version without the watermark The stress-strain curve is given in fig. 4 . The experimental strength values obtained from a unidirectional coupon test [21] are 11\% lower than the predicted value. While this is an acceptable error, the failure criterion parameters should be recalibrated to reduce further it.

Selected stress contours are given in fig. 5. The kink band width, $w$ was predicted to be $180 \mu \mathrm{m}$, which is within the range observed experimentally (assuming fiber diameter of $8 \mu \mathrm{m}$ ). The width is determined by the current material stiffness and the failure criterion parameters and therefore cannot be calibrated independently (if one wishes to closely match the experimental data). 


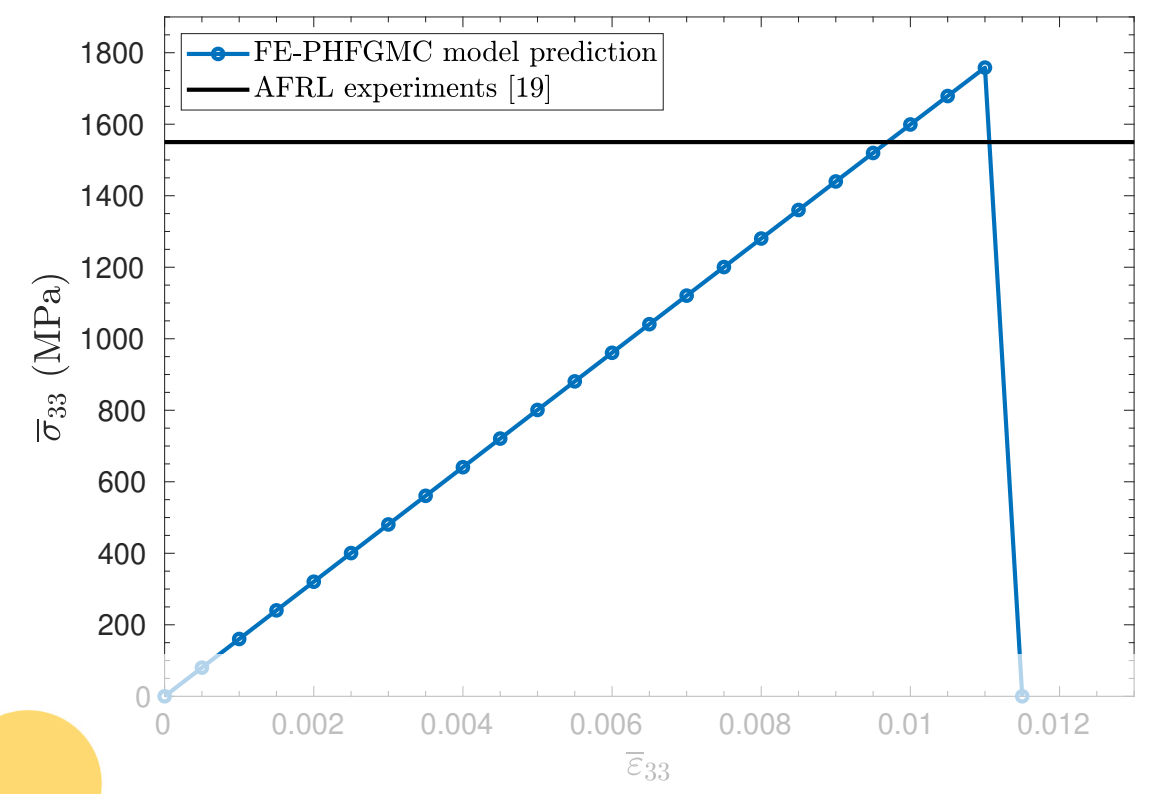

Figure 4: The FE-PHFGMC prediction for the axial response of the mesoscale problem under compression and the strength is compared to experimental value [21]
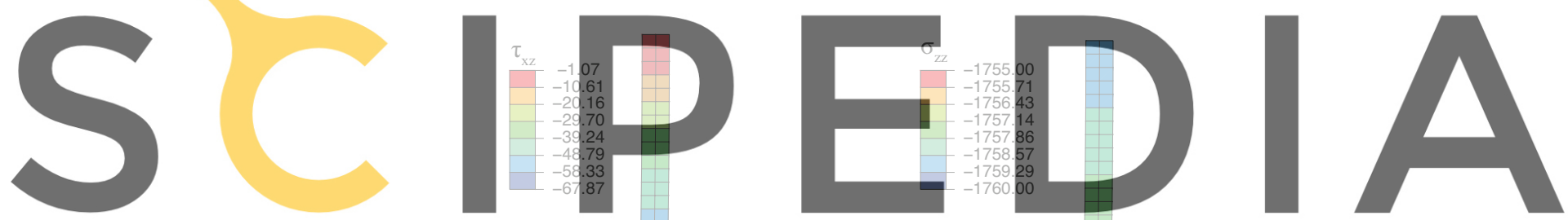

Register for free at https//www.scipedia.côm to download the version without the watermark
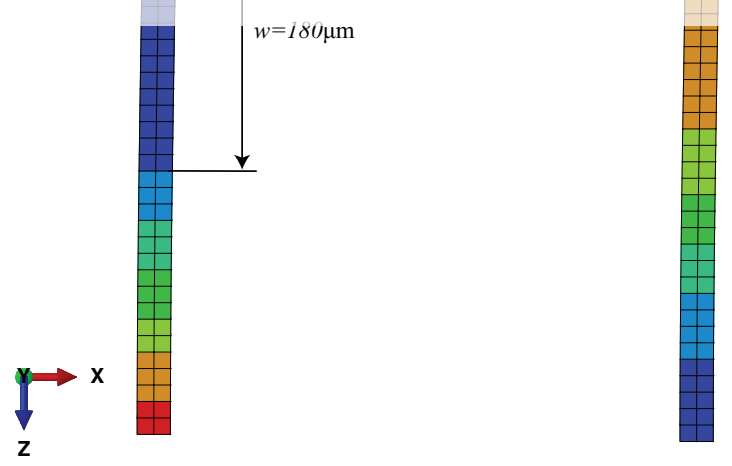

Figure 5: The FE-PHFGMC predictions of the macroscopic shear and axial stress fields (left and right, respectively) in the mesoscale problem under compression. The kink band width, $w$ is within the range observed in experiments. 
It was found that the matrix nonlinear constitutive law alone was sufficient to generate the instability, but the kink band width prediction was incorrect as it kept growing in size after the kink band had formed. The failure criterion was added to control the width, which is otherwise determined by the fiber diameter [5] that is not explicitly modeled here (it was homogenized).

\section{Acknowledgement}

Acknowledgement This research was supported by the Israel Science Foundation(ISF) (grant No. 2314/19). The last author gratefully acknowledges the support of the Nathan Cummings Chair in Mechanics.

\section{REFERENCES}

[1] Breiman, U., Aboudi, J. \& Haj-Ali, R. Semianalytical compressive strength criteria for unidirectional composites. Journal of Reinforced Plastics and Composites 37, 238-246 (2018). URL http://journals.sagepub.com/doi/10.1177/0731684417740982.

[2] Joannie W. Chin. Composite Materials - Strengthening Infrastructure Development (2018). URL https://www.nist.gov/speech-testimony/ composite-materials-strengthening-infrastructure-development.

[3] Rosen, B. Mechanics of composite strengthening. (1965). URL https://ntrs.nasa.gov/ search. jsp?R=19660035520.

[4] Argon, A. S. Fracture of Composites. Treatise on Materials Science and Technology 1, 79-114
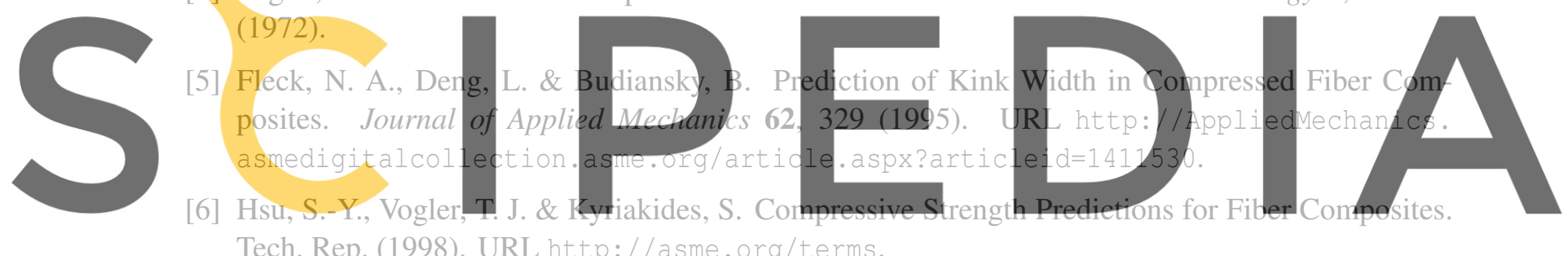

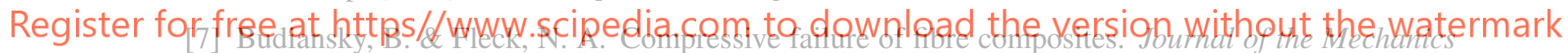
and Physics of Solids 41, 183-211 (1993).

[8] Vogler, T. J. \& Kyriakides, S. On the axial propagation of kink bands in fiber composites : Part i experiments. International Journal of Solids and Structures 36, 557-574 (1999). URL http: //www.sciencedirect.com/science/article/pii/s0020768398000298.

[9] Naya, F. et al. Computational micromechanics of fiber kinking in unidirectional FRP under different environmental conditions. Composites Science and Technology 144, 26-35 (2017). URL https: //www.sciencedirect.com/science/article/pii/s0266353817300416.

[10] Vogler, T. J., Hsu, S.-Y. \& Kyriakides, S. On the initiation and growth of kink bands in ®ber composites. Part II: analysis. Tech. Rep. URL www.elsevier.com/locate/i jsolstr.

[11] Takahashi, T., Ueda, M., Iizuka, K., Yoshimura, A. \& Yokozeki, T. Simulation on kink-band formation during axial compression of a unidirectional carbon fiber-reinforced plastic constructed by X-ray computed tomography images. Advanced Composite Materials 28, 347-363 (2019). URL https://www.tandfonline.com/doi/full/10.1080/09243046.2018.1555387. 
[12] Haj-Ali, R. \& Aboudi, J. A new and general formulation of the parametric HFGMC micromechanical method for two and three-dimensional multi-phase composites. International Journal of Solids and Structures 50, 907-919 (2013). URL http://www.sciencedirect.com/science/ article/pii/S0020768312004726.

[13] Haj-Ali, R., Zemer, H., El-Hajjar, R. \& Aboudi, J. Piezoresistive fiber-reinforced composites: A coupled nonlinear micromechanical-microelectrical modeling approach. International Journal of Solids and Structures 51, 491-503 (2014). URL http://dx.doi.org/10.1016/j.ijsolstr. 2013.10.022.

[14] Levi-Sasson, A., Aboudi, J., Matzenmiller, A. \& Haj-Ali, R. Failure envelopes for laminated composites by the parametric HFGMC micromechanical framework. Composite Structures 140, 378-389 (2016). URL http://dx.doi.org/10.1016/j.compstruct.2015.12.035.

[15] Haj-Ali, R. \& Aboudi, J. The Parametric HFGMC Micromechanics. In Micromechanics and Nanomechanics of Composite Solids, 391-424 (Springer International Publishing, Cham, 2018). URL http://link.springer.com/10.1007/978-3-319-52794-9_14.

[16] Haj-Ali, R. \& Aboudi, J. Nonlinear micromechanical formulation of the high fidelity generalized method of cells. Int. J. of Solids and Struct. 46, 2577 - 2592 (2009). URL http://www.sciencedirect.com/science/article/pii/s002076830900071710. 1016/j.ijsolstr.2009.02.004https://ac.els-cdn.com/S0020768309000717/1-s2. 0-S0020768309000717-main.pdf?_tid=3c2ace36-9c95-48c1-9fa3-aa1b0cb4f574\&

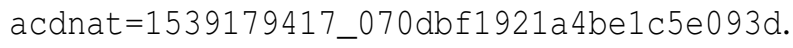

[17] Abaqus Documentation. ORIENT . URL https://abaqus-docs.mit.edu/2017/English/ SIMACAESUBRefMap/simasub-c-orient.htm.

[18] Ramberg, W. \& Osgood, W. R. Description of stress-strain curves by three parameters. National Advisory Committee for Aeronautics Technical Note No. 902 (1943). URL http: / hdl . handle. net/2060/19930081614.

[19] Abaqus Documentation. Generalization to multiaxial stress states. URL https://abaqus-docs . mit.edu/2017/English/SIMACAEMATRefMap/simamat-c-deformationplast.htm\# simamat-c-deformationplast-t-GeneralizationToMultiaxialStressStates-sma-topic2.

[20] Lo, K. \& Chim, E.-M. Compressive Strength of Unidirectional Composites. Journal of Reinforced Plastics and Composites 11, 838-896 (1992). URL http:// journals.sagepub.com/doi/10. $1177 / 073168449201100801$.

[21] Clay, S. B. \& Knoth, P. M. Experimental results of quasi-static testing for calibration and validation of composite progressive damage analysis methods URL https: / / journals. sagepub.com/doi/ $\mathrm{pdf} / 10.1177 / 0021998316658539$. 